\title{
Comparison of LED Emitting and Fluorescent Lighting on the Stress Evoked Cerebral Dysfunctions and Electric Activity of the Brain
}

\author{
Fedor M Kiryukhin', Olga E Zheleznikova ${ }^{2}$, Dmitriy A Labunskiy ${ }^{3 *}$, Vyacheslav G Podsevatkin ${ }^{4}$ and Svetlana V Kiryukhina ${ }^{5}$ \\ 1,2,3,4,5 NP Ogarev Mordovia State University, Departments of Neurology \\ and Psychiatry, and Light Technology, Saransk, Russia \\ ${ }^{*}$ Corresponding author \\ Dmitriy A Labunskiy, NP Ogarev Mordovia State University, Departments \\ of Neurology and Psychiatry, and Light Technology, Saransk, Russia \\ Submitted: 25 May 2020; Accepted: 05 Jun 2020; Published: 20 Jun 2020
}

\begin{abstract}
The study of the effect of LED lighting on the physiological functions of the body takes on a special role, taking into account the already proven facts of the effectiveness of such effects primarily on the organ of vision, especially on the retina, skin condition and various mechanisms of protein metabolism, as well as the activity of expression of certain genes. In our study, an attempt was made to study the role of this radiation on the main indicators of brain function under stress, especially the nature of changes in its bioelectric activity. It seems to us that this study will help to better understand the work of this key human organ in conditions of protective light-emitting radiation under various stress effects.
\end{abstract}

\section{Introduction}

With various pathological conditions of the body, the EEG components change in frequency, amplitude, shape. EEG indices even in healthy people are very variable. The nature of the EEG may vary depending on the functional state of the brain. After the negative impact of the external environment, combined with rapid eye fatigue, a decrease in concentration of attention and memory, signs of emotional-psychological arousal with the appearance of fast rhythms are recorded on the EEG, which must be taken into account when developing a comprehensive assessment of the effect of LED lighting on the human body

\section{Materials and Methods}

The following types of studies were carried out: the study of mental functions on the cards of Avrutsky-Zaitsev, Schulte tables; ECG examination; EEG study of the functional activity of the brain; ECHO-EG study of the brain. Selected observers in the amount of 60 people were randomly divided into two groups of 30 people in each: control (experiment with illumination of fluorescent lighting) and the main (experiment with LED application). The number of observers and the number of experiments was determined on the basis of the requirements for obtaining statistically reliable data and limiting the time of research. Work schedules for each group of observers were compiled. Each observer participated in the experiment only at the same time, taking into account human daily biorhythms.

\section{Results}

EEG studies of brain parameters were performed for the control and main groups before and after the series of experiments (on the 1 st and 60th day). To study the mechanisms of changing the functional parameters of the brain, groups of observers were additionally tested on the 30th day of research.

It was revealed that in the control group on the 1st day of the study, the EEG data were within the limits of acceptable standards, while further studies (on the 30th and 60th day of the experiment) showed changes in EEG indicators. In tables1 and 2, the dynamics of the EEG alpha rhythm indices (index, frequency, amplitude) for the control group is given as an example. The alpha rhythm is characterized by several parameters, but the most important are its amplitude and frequency. For all observers, tables of changes in time of the amplitude and frequency of the spectral peak were constructed.

As follows from Figure 1 alpha activity is almost not expressed. Groups of slow waves of medium amplitude are recorded. 


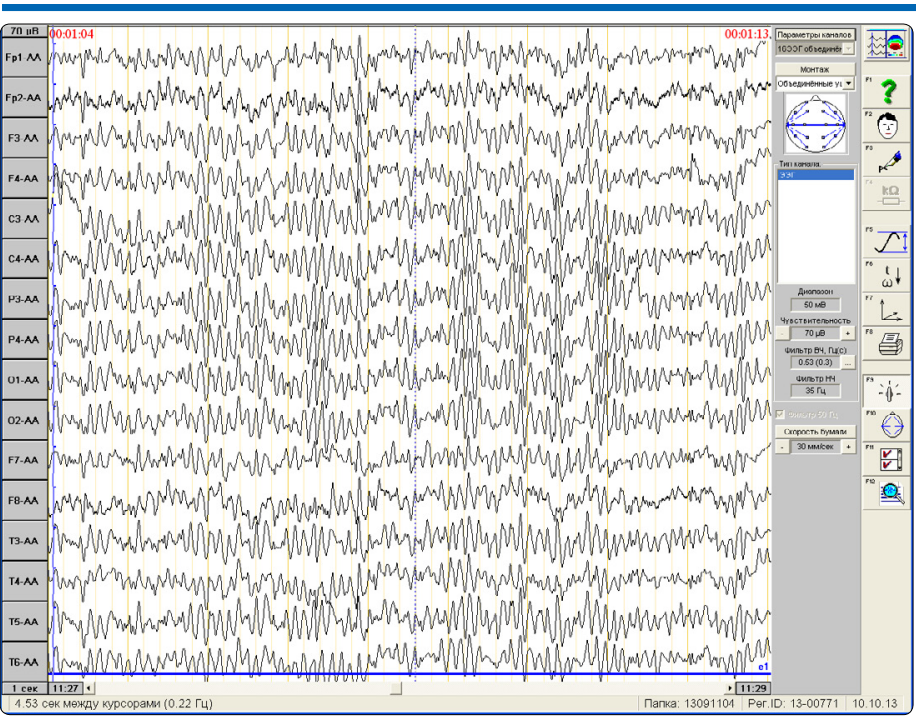

Figure 1: EEG of observer U. from the main group on the 1st day

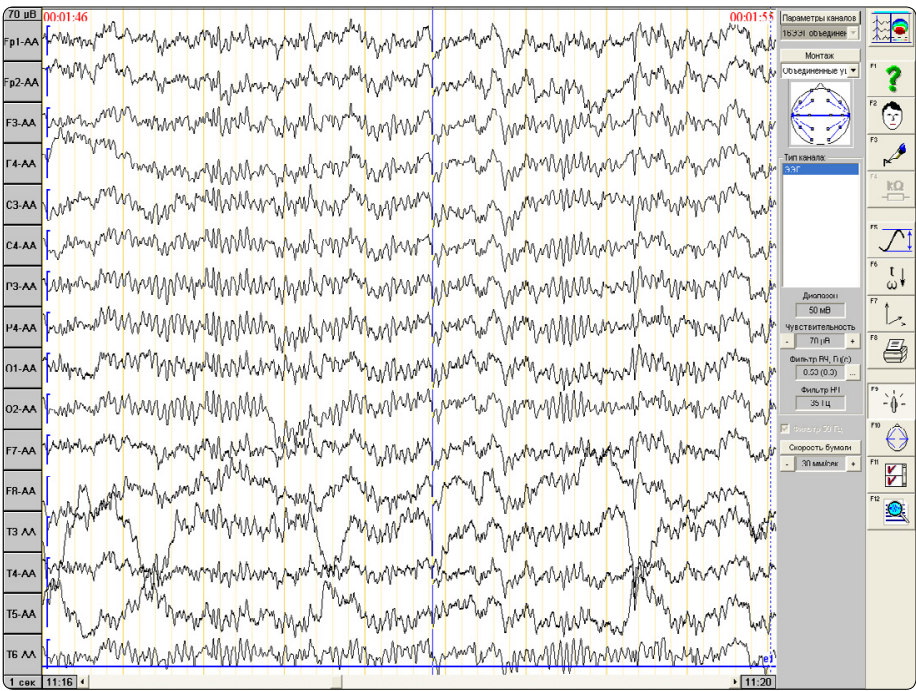

Figure 2: EEG of observer U. from the main group on the 30st day

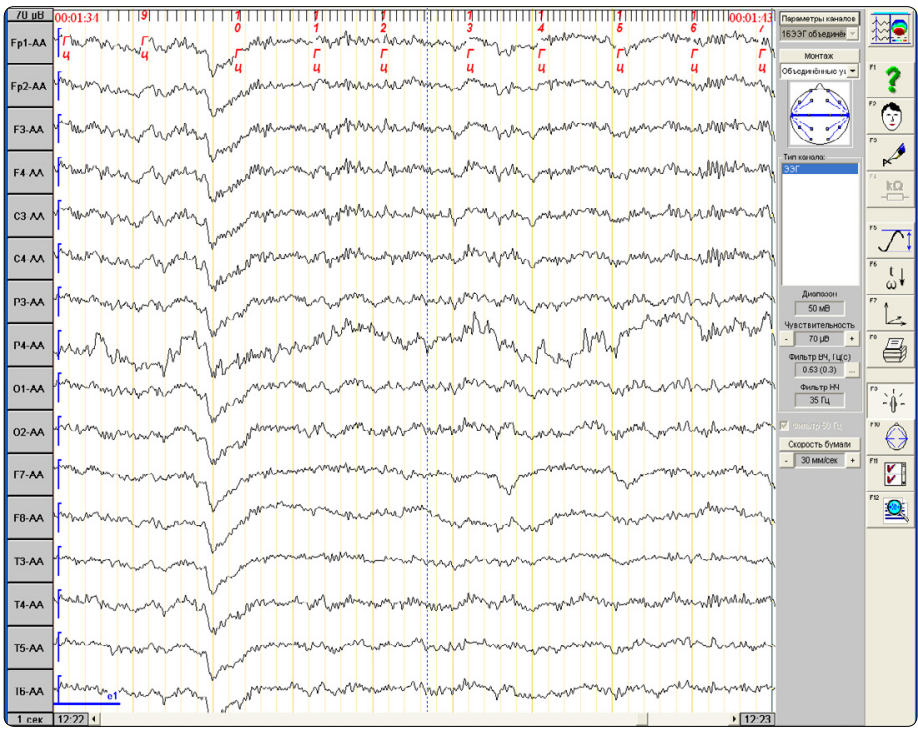

Figure 3: EEG of observer $U$. from the main group on the 60st day
The EEG results for observers of the main group working in the conditions of illumination of diabetes mellitus on the 1st day of the study showed that the EEG indicators are within the acceptable range of norms. With further exposure to LED lighting (on the 30th and 60th days), the dynamics of the amplitude of the alpha rhythm in the central parietal and occipital regions is noted - it has a smoothly declining character. Slight changes in the amplitude of the spectral peak were observed.

[Tables 1] show, as an example, the dynamics of the EEG alpha rhythm indices (index, frequency, amplitude) for the main group working under the conditions of LED illumination. For all observers, tables of changes in time of the amplitude and frequency of the spectral peak were constructed.

Table 1: The dynamics of the index of the EEG alpha rhythm of the control group observers (lighting with fluorescent lamps)

\begin{tabular}{|l|l|l|l|}
\hline \multicolumn{5}{|l|}{ Alpha rhythm analysis 8.0 - 13.0 Hz } \\
\hline Index & $\begin{array}{l}\text { Index\% } \\
\text { 1st day }\end{array}$ & $\begin{array}{l}\text { Index } \% \\
\text { 30th day }\end{array}$ & $\begin{array}{l}\text { Index\% } \\
\text { 60th day }\end{array}$ \\
\hline Fp1 & $45,80 \pm 3,18$ & $34,63 \pm 2,92^{*}$ & $32,70 \pm 2,87^{*}$ \\
\hline Fp2 & $38,20 \pm 3,50$ & $30,47 \pm 2,49^{*}$ & $28,63 \pm 2,52$ \\
\hline F3 & $55,47 \pm 4,85$ & $34,50 \pm 3,05^{*}$ & $29,50 \pm 2,89^{*}$ \\
\hline F4 & $55,33 \pm 4,61$ & $32,63 \pm 3,22^{*}$ & $28,53 \pm 3,11^{*}$ \\
\hline C3 & $63,20 \pm 3,73$ & $44,67 \pm 4,50^{*}$ & $41,62 \pm 4,28^{*}$ \\
\hline C4 & $59,73 \pm 3,41$ & $43,13 \pm 3,90^{*}$ & $39,38 \pm 3,17^{*}$ \\
\hline P3 & $64,57 \pm 3,14$ & $41,50 \pm 4,00^{*}$ & $38,50 \pm 3,97^{*}$ \\
\hline P4 & $58,13 \pm 4,63$ & $38,30 \pm 3,11^{*}$ & $36,12 \pm 3,29^{*}$ \\
\hline O1 & $63,37 \pm 3,08$ & $40,50 \pm 3,26^{*}$ & $35,53 \pm 3,02^{*}$ \\
\hline O2 & $69,37 \pm 2,21$ & $46,27 \pm 4,15^{*}$ & $41,93 \pm 4,51^{*}$ \\
\hline F7 & $35,20 \pm 4,34$ & $22,33 \pm 1,85^{*}$ & $19,33 \pm 1,68^{*}$ \\
\hline F8 & $36,83 \pm 4,60$ & $24,93 \pm 3,00^{*}$ & $22,86 \pm 2,84^{*}$ \\
\hline T3 & $36,97 \pm 5,04$ & $20,47 \pm 2,69^{*}$ & $17,33 \pm 2,03^{*}$ \\
\hline T4 & $37,83 \pm 4,17$ & $21,50 \pm 2,78^{*}$ & $18,20 \pm 2,13^{*}$ \\
\hline T5 & $44,67 \pm 6,15$ & $23,37 \pm 3,30$ & $20,75 \pm 3,43$ \\
\hline T6 & $39,57 \pm 5,87$ & $20,10 \pm 2,78^{*}$ & $19,12 \pm 2,14^{*}$ \\
\hline No $*$ & & & \\
\hline
\end{tabular}

Note: ${ }^{*}$ differences from the corresponding values on the first day are significant at $\mathrm{p}<0.05$.

Table 2: The dynamics of the frequency of the EEG alpha rhythm of the control group observers (lighting with fluorescent lamps)

\begin{tabular}{|l|l|l|l|}
\hline Alpha rhythm analysis 8.0-13.0 Hz & \multicolumn{3}{l|}{} \\
\hline Index & $\begin{array}{l}\text { Frequency Hz } \\
\text { 1st day }\end{array}$ & $\begin{array}{l}\text { Frequency Hz } \\
\text { 30st day }\end{array}$ & $\begin{array}{l}\text { Frequency Hz } \\
\text { 60st day }\end{array}$ \\
\hline 1 & 2 & 3 & 4 \\
\hline Fp1 & $10,36 \pm 0,06$ & $10,37 \pm 0,07$ & $10,39 \pm 0,08$ \\
\hline Fp2 & $10,39 \pm 0,07$ & $10,41 \pm 0,07$ & $10,43 \pm 0,08$ \\
\hline F3 & $10,41 \pm 0,07$ & $10,36 \pm 0,08$ & $10,34 \pm 0,07$ \\
\hline F4 & $10,37 \pm 0,07$ & $10,41 \pm 0,09$ & $10,42 \pm 0,08$ \\
\hline C3 & $10,41 \pm 0,08$ & $10,38 \pm 0,10$ & $10,36 \pm 0,09$ \\
\hline C4 & $10,34 \pm 0,08$ & $10,37 \pm 0,09$ & $10,39 \pm 0,09$ \\
\hline P3 & $10,43 \pm 0,08$ & $10,41 \pm 0,09$ & $10,38 \pm 0,07$ \\
\hline P4 & $10,45 \pm 0,09$ & $10,47 \pm 0,10$ & $10,49 \pm 0,09$ \\
\hline
\end{tabular}

End of table 2. 


\begin{tabular}{|l|l|l|l|}
\hline 1 & 2 & 3 & 4 \\
\hline O1 & $10,48 \pm 0,11$ & $10,49 \pm 0,11$ & $10,51 \pm 0,12$ \\
\hline O2 & $10,46 \pm 0,10$ & $10,52 \pm 0,11$ & $10,56 \pm 0,12$ \\
\hline F7 & $10,44 \pm 0,06$ & $10,46 \pm 0,07$ & $10,47 \pm 0,08$ \\
\hline F8 & $10,34 \pm 0,07$ & $10,36 \pm 0,07$ & $10,37 \pm 0,06$ \\
\hline T3 & $10,44 \pm 0,07$ & $10,44 \pm 0,08$ & $10,44 \pm 0,08$ \\
\hline T4 & $10,38 \pm 0,07$ & $10,44 \pm 0,08$ & $10,48 \pm 0,09$ \\
\hline T5 & $10,39 \pm 0,08$ & $10,40 \pm 0,09$ & $10,43 \pm 0,08$ \\
\hline T6 & $10,40 \pm 0,08$ & $10,48 \pm 0,10$ & $10,53 \pm 0,11$ \\
\hline
\end{tabular}

Note: * differences are significant at $\mathrm{p}<0.05$.

\section{Conclusion}

The analysis of the results of tables 1 and 2 shows that in the main group there are insignificant changes in the amplitude of the spectral peak, which can be regarded as an acceptable version of the norm. The dynamics of the amplitude of the alpha rhythm in the central parietal and occipital regions has a smoothly declining character.

An analysis of the amplitudes of the beta and theta rhythms on the EEG of observers from the main group working in conditions of illumination of diabetes revealed that the amplitude on the 30th and 60th days remains unchanged compared to the 1 st.

When analyzing EEG indices on the background EEG recording, it was noted that alpha activity - a high index with moderately expressed alpha rhythm modulations - does not change under the influence of illumination of diabetes. Figures 1, 2 and 3 show examples of EEG of an observer from the main group on different days of the examination.
A decrease in the spectral peak dynamics under the influence of fluorescent lighting on the 30th and, to a greater extent, on the 60th days of observation, with normal parameters of this indicator when using LED light sources, indicates a less favorable effect of fluorescent lighting on the neurophysiological parameters of the brain and indicates a decrease in functional activity bioelectric potentials.

Thus, a comprehensive study of electrophysiological functions under the influence of various types of lighting allows us to develop criteria for evaluating the favorable and adverse effects of light sources on the human body, as well as highlight electrophysiological markers of the adverse functioning of the nervous system. Creating human life safety in the environment requires additional methods to study the effects of various light sources on the psychosomatic functions of the body [1-3].

\section{References}

1. Rahman SA, St Hilaire MA, Lockley SW (2017) The Effects of Spectral Tuning of Evening Ambient Light on Melatonin Suppression, Alertness and Sleep. Physiol Behav 177: 221-229.

2. Cajochen C, Frey S, Anders D, Späti J, Bues M, et al. (1985) Evening exposure to a light-emitting diodes (LED)-backlit computer screen affects circadian physiology and cognitive performance. J Appl Physiol 110: 1432-1438.

3. Niemierzycka A, Tomczuk K, Mikicin M, Zdrodowska A, Orzechowski G, et al. (2018) Examinations of the methods used to power supply of different light sources and their effect on bioelectrical brain activity. Neurol Neurochir Pol 52: 505-513.
Copyright: (02020 Dmitriy A Labunskiy, et al. This is an open-access article distributed under the terms of the Creative Commons Attribution License, which permits unrestricted use, distribution, and reproduction in any medium, provided the original author and source are credited. 